\title{
Self-compensating fiber optic flow sensor system and its field applications
}

\author{
Wei Peng, Gary R. Pickrell, Zhengyu Huang, Juncheng Xu, Dae Woong Kim, \\ Bing Qi, and Anbo Wang
}

\begin{abstract}
A self-compensating fiber optic flow sensor system based on the principle of broadband white-light interferometers and cantilever beam bending is described. The fiber optic sensor system uses two fiber ferrule sensors that are bonded on either side of a cantilever beam to measure the flow rate by monitoring the air-gap changes caused by the bending of the cantilever beam. Cross sensitivity of the temperature and pressure dependence of the sensor can be compensated for automatically. The prototype sensor system was constructed, laboratory characterized, and field tested. The results from the field testing have demonstrated high resolution, repeatability, and stability for on-line detection of the flow rates of fluids. (C) 2004 Optical Society of America
\end{abstract}

OCIS codes: $\quad 060.2310,060.2370,120.3180$.

\section{Introduction}

Flow rate is one of the key measurement parameters for both the down-hole oil and the surface piping systems of the oil production industry. Real-time, on-line monitoring of single-phase or multiphase fluid flow rates over a large dynamic range with high accuracy, good reliability, and cost effectiveness is important for realizing the full economic potential of the petroleum industry. ${ }^{1,2}$ Usually, conventional single-phase fluid flowmeters with electric signal outputs are operated by the combination of electrical sensing elements and mechanical devices. The fluid flow is monitored by detection of changes in a parameter such as frequency of turbine, frequency of vortexshedding-induced vibration, flow-induced pressure, or flow-induced differential temperature.,4 Owing to the harsh environment in oil fields, however, including high temperature, high pressure, and presence of corrosive agents, electrical flowmeters such as paddlewheel flowmeters, turbine flowmeters,

The authors are with the Center for Photonics Technology, Bradley Department of Electrical and Computer Engineering, Virginia Polytechnic Institute \& State University, Suite 303, 460 Turner Street, Blacksburg, Virginia 24061-0287. W. Peng's e-mail address is weipeng@vt.edu.

Received 14 April 2003; revised manuscript received 24 October 2003; accepted 12 December 2003.

0003-6935/04/081752-09\$15.00/0

(C) 2004 Optical Society of America vibration-based flowmeters, and Pitot-tube flowmeters are not suitable for applications there.5,6

Because of the many inherent advantages of fiberoptic sensors, fiber optic flow sensors have been investigated extensively in the past two decades. Generally, most fiber flow sensors can be classified into three groups, although in some cases the technology may fit into more than one group.

Vortex-shedding-based fiber optic flow sensors ${ }^{7-9}$ use the optical fiber as the bluff-body sensing element whose oscillation frequency is affected by vortex shedding. The low photoelastic coefficient of silica glass fiber ${ }^{10}$ limits the sensitivity of this kind of sensor. Therefore a long sensing fiber is necessary to produce the degree of sensitivity that makes the sensor extremely thermally unstable. Also, the fragile fiber must be positioned through the cross-sectional areas of the pipe, making the fiber difficult to deploy.

The second group comprises laser Doppler velocimeters, ${ }^{11,12}$ which are based on the detection of the Doppler frequency shift of scattering waves caused by moving particles in the flow. This type of flow sensor cannot be used for a fluid with a high particle density. These sensors usually are bulky and have a complex signal-processing system.

The third group consists of interferometrically based fiber flow sensors in which the low-coherence or speckle spectrum of temporally or randomly fluctuating light interference signals ${ }^{13,14}$ has been modulated for flow detection. Only the relative velocities are obtained with this method. Also, the velocity distribution along the depth cannot in principle be 


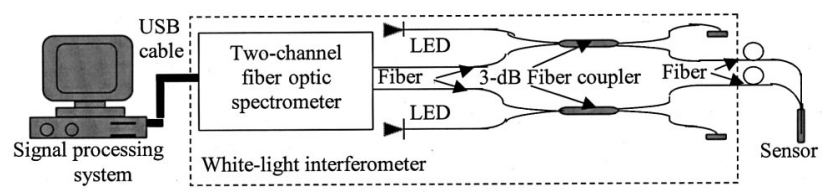

Fig. 1. Schematic diagram of the fiber optical flow sensor system.

measured because additional traversing equipment is needed and all scattering components along the depth contribute to the formation of a speckle pattern. Also included in this group are the broadband interferometric flow sensors ${ }^{15,16}$ that can be limited by thermal and pressure instabilities caused by the environment. Additionally, photon correlation spectroscopy, which is more suitable for biotechnology applications, has been used to measure particle size and flow velocity ${ }^{17}$; surface acoustic wave and shear horizontal acoustic plate mode oscillation techniques were proposed for liquid or microliquid flow measurement, ${ }^{18}$ and the related electrical or optomechanical devices add to the complexity of the sensor system. Also, Dunkers et al. ${ }^{19}$ described using a long-period grating fiber optic flow sensor to detect resin flow in composite manufacture.

For all the groups of fiber optic flow monitoring sensors mentioned above, there often are obstacles to sensor deployment in the oil field. Still, the principal advantages of tubing-based fiber optic interferometric sensors are well established and have been demonstrated to be effective for high-sensitivity and high-accuracy measurements of a variety of other physical parameters such as temperature, pressure, and strain. ${ }^{20,21}$ Thus these sensors are attractive for use in the design of flow sensors as well. To overcome the especially harsh conditions of the down-hole oil environment and its unique requirements with respect to temperature sensitivity, pressure isolation, sensitivity to high flow rate, and large dynamic range, a self-compensating fiber optic interferometric flow sensor system has been designed and developed. Its interferometric signal demodulation results in a unique combination of high resolution, selfcompensation for temperature and pressure sensitivity, and compactness of structure as well as simplification of deployment. A prototype fiberoptic flow sensor system was developed and tested both in the lab and in the field; the test results have demonstrated that this fiber optic flow sensor system can detect flow rates of fluids with high degrees of resolution, reliability, and stability.

\section{Operational Principle of the Self-Compensation Technique}

The self-compensating fiber optic flow sensor is illustrated schematically in Fig. 1. The system is composed of a sensor probe, a fiber spectrometer for optoelectronic signal processing, ${ }^{22}$ and a dual multimode fiber cable linking the sensor probe and the signal-processing unit.

The broadband light from two light-emitting diodes (LEDs) is launched into two 3-dB 50:50 multimode

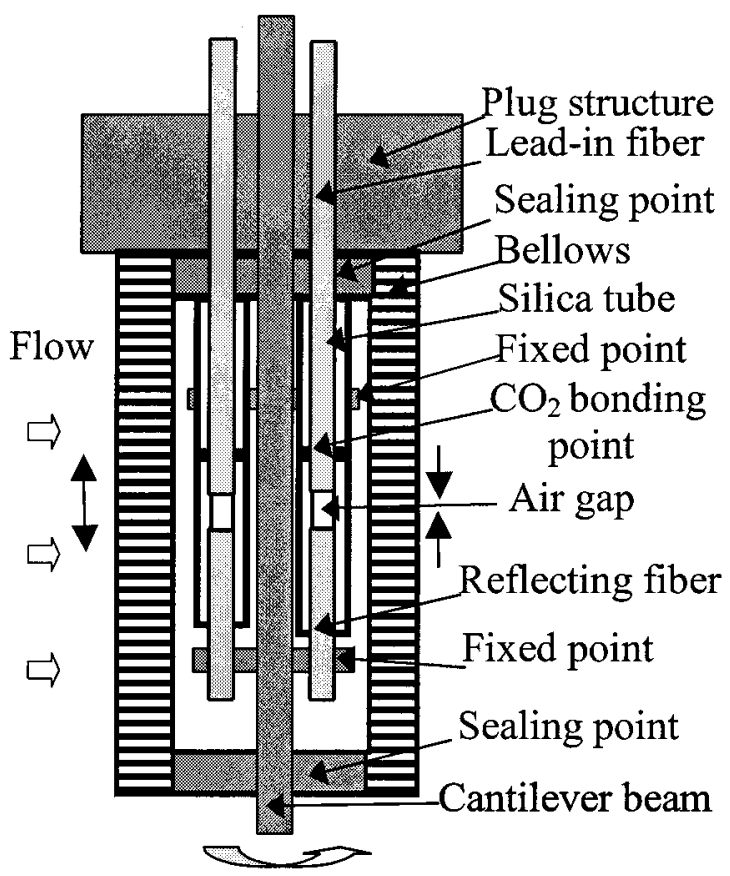

Fig. 2. Illustration of the fiber optical flow sensor head.

fiber couplers, which split each beam into two equal signals. One signal propagates along the lead-in fiber to the sensor head, and the other terminates at the end face of one of the coupler's output fibers. The signal reflected from the sensor head propagates along the same fiber to the same coupler, and then both of these light beams are detected by the spectrometer. The detected spectra are analyzed by an algorithm designed for white-light interferometry. The way in which the signal-demodulation system functions was described thoroughly in Ref. 22 and so will not be repeated here. The flow rate can be demodulated from the detected light signal fringes as described below.

The configuration of the fiber optic flow sensor head, as shown in Fig. 2, is a combination of the broadband white-light interferometer and cantileverbeam designs. Based on the strain principle of cantilever beams, ${ }^{23}$ one can determine the flow rate by detecting the changes in air gap of the two fiber sensors caused by the bending of the cantilever beam. The sensor head is fabricated by bonding of two identical fiber optic tube-based sensors on a cantilever beam. Each sensor is fabricated by insertion of two fibers into a fused-silica hollow glass tube. The process is somewhat different from normal tube-based fiber optic sensor fabrication ${ }^{21}$ because, whereas one end of a capillary is thermally bonded to the lead-in fiber, the reflecting fiber is left free to move in the other end of tube. The process is repeated for the other side of the beam, such that a sensor is fabricated on each side. Both ends of the tube and both reflecting fibers of each of the two sensors are firmly bonded to the metal cantilever beam at a predetermined gauge length, which allows the air gap to change freely without restriction by the capillary 
tube. This dual-sensor combination is then inserted into the central hole of a metal tube or plug, which permits easy installation of the sensor plug into a flow pipe for measurement. Finally, a small metal bellows is used to cover the beam and the sensors. This bellows allows the flow to bend the cantilever beam located inside the pipe without allowing the fluid to come into contact with the cantilever beam and the sensors. In this manner the sensor can be isolated from fluids in the outside environment. When a fluid flows past the sensor, the cantilever beam will be bent by the momentum of the fluid passing through the beam. The end of the beam protruding from the bellows will bend with the applied flow; the amount of deflection of the beam was small for the flow rates tested. Therefore, for clarity, bending of the tip of the beam is not shown in Fig. 2.

When fluids impinges upon the beam, all momentum of the impinging fluid to the sensor area can be converted into force; then the force on the beam can be expressed as

$$
F t=m v,
$$

where $F$ is the force on the cantilever, $t$ is the unit of time, $m$ is the mass of the fluid that passes the sensor area within the unit time, and $v$ is the velocity of the fluid. If an incompressible fluid element is uniformly decelerated from velocity $v$ to zero in time $t$, its average velocity is $v / 2$. We can have

$$
\begin{aligned}
m & =\rho V, \\
V & =(1 / 2) S v t, \\
S & =c L_{b},
\end{aligned}
$$

where $\rho$ is the density of the fluid, $V$ is the volume of flow in unit time, $S$ is the cross-sectional area of the cantilever beam seen by the fluid, $c$ is the width of the cantilever, $d$ is thickness of the cantilever, and $L_{b}$ is the length of the cantilever beam. From Eqs. (1) and (2), the applied force is

$$
F=\rho c L_{b} v^{2} / 2 .
$$

Assume that $F$ is a uniform force; there will be maximum strain $\varepsilon$ at the fixed end of the cantilever ${ }^{23}$ as follows:

$$
\varepsilon=\frac{6 F L_{b}}{E c d^{2}}
$$

where $E$ is Young's modulus. When the flow passes by, because each of the sensor's free fibers is bonded with the cantilever, the bending of the cantilever beam will be manifested in an air-gap change. The air-gap change caused by the gauge-length change between the two bonding points $\Delta L$ is represented as

$$
\Delta L=\varepsilon L,
$$

where $L$ is the gauge length between the two bonding points. Then, from Eqs. (2)-(5), we get

$$
\Delta L=\frac{3 \rho v^{2} L_{b}^{2} L}{E d^{2}}=\beta(v),
$$

where $\beta$ denotes the relationship between air-gap change $\Delta L$ and the velocity of the flow, $v$.

When the cantilever beam bends because of the flow of the fluid, the air gap of the sensor that faces in the direction of the flow of the fluid will increase while the air gap of the sensor located on the opposite side of the cantilever beam will decrease. For a given cantilever material, the change in the ratio of thermal expansion coefficient to compressibility is negligible within normal ranges of environmental temperature and pressure. Therefore, when the fluid passes by the sensor, the air gaps of both sensors will change but in opposite directions. However, the two sensors will have the same magnitude and direction of change in air gap with respect to the temperature and pressure changes:

$$
\begin{aligned}
& \Delta L_{1}=\alpha_{1} \Delta T+K \alpha_{1} \Delta P+\beta_{1}(v), \\
& \Delta L_{2}=\alpha_{2} \Delta T+K \alpha_{2} \Delta P+\beta_{2}(v),
\end{aligned}
$$

where $\Delta L_{1}$ and $\Delta L_{2}$ represent the gauge-length changes of the two sensors, $\alpha_{1}$ and $\alpha_{2}$ are the temperature effects of the two sensors, $K$ is the ratio of the effects of thermal extension and pressure extension, and $\beta_{1}$ and $\beta_{2}$ refer to the flow coefficients of the two sensors as shown in Eq. (6). Based on Eq. (7), we get

$$
F(v)=\alpha_{2} \Delta L_{1}-\alpha_{1} \Delta L_{2}=\alpha_{2} \beta_{1}(v)-\alpha_{1} \beta_{2}(v),
$$

where $F$ is the relationship of flow velocity $v$ and $\Delta L_{1}$ and $\Delta L_{2}$, the gauge-length changes of the two sensors. From Eq. (8) we can see that by analyzing the outputs of the two sensors this sensor system can detect the applied flow velocity while remaining insensitive to pressure and temperature changes.

If we select a brass cantilever beam, with $E=4.2 \times$ $10^{10} \mathrm{~kg} / \mathrm{m}^{2}, L_{b}=25 \mathrm{~mm}, c=5 \mathrm{~mm}$, and $d=0.5 \mathrm{~mm}$, two fiber sensors with gauge length $L=20 \mathrm{~mm}$, and an original air gap $a=12.00 \mu \mathrm{m}$, the relationship between the air-gap changes and the applied flow velocity will be as shown in Fig. 3 . It can be seen that the air gaps of both the front and the back sensors are affected by temperature changes. However, because one sensor will experience an air-gap increase and the other sensor will experience an air-gap decrease with temperature changes, the effects of temperature can be canceled by calculation. The figure illustrates that, with the dual-sensor structure, this flow sensor design can compensate automatically for changes in the outside temperature through calculation of the temperature effect; thus the output will be related only to the applied flow rate.

We used the cantilever-beam flow-induced bending calculation as an approximation to our system, which 


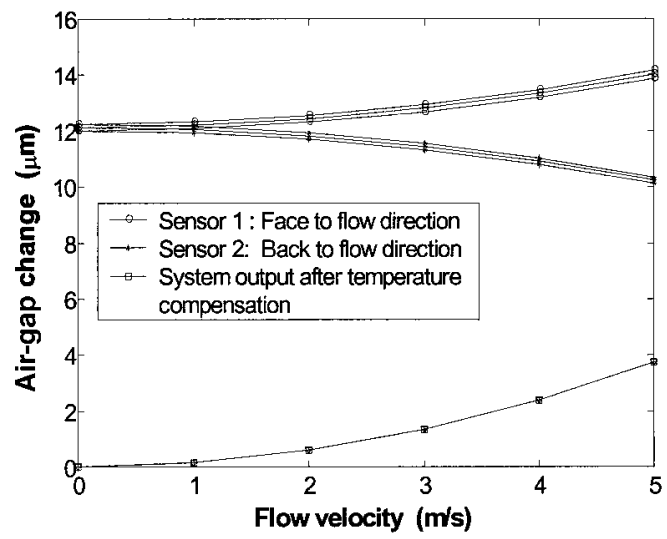

Fig. 3. Result of simulation of a self-compensating fiber optic flow sensor.

contains a round bellows that covers the beam. The round bellows will change the flow deflection slightly, but the overall change will be small relative to the measured flow rates desired. In industrial practice, flow rate rather than flow velocity is usually used for measuring flow; one needs to consider the crosssectional area of the flow pipe to change flow velocity to flow rate in Eq. (8). Because the flow rate depends on the area-integrated velocity profile, and because generally the velocity profile across the pipe will not be uniform, the calibration will depend on the sensor's position within the pipe.

\section{Experimental Procedures and Lab Testing}

The prototype flow sensor system was constructed according to design described above. Two Honeywell 4854 LEDs with a center wavelength of $\sim 850 \mathrm{~nm}$ pigtailed with standard 50-125- $\mu \mathrm{m}$ multimode fibers were used as broadband light sources. We used an Ocean Optics, Inc., PC-2000 dual-channel fiber optic spectrometer to detect the optical signals from the sensor head, and its output was fed to the flow-rate measurement and control system based on a highresolution white-light interferometer. ${ }^{22}$

The analysis described in Section 2 provides a clear guideline to the design of the fiber optic flow sensor. We improved the fabrication technique sequentially by making many sensor heads. Because of the low thermal expansion coefficient of $1.0 \times 10^{-6} /{ }^{\circ} \mathrm{C}$ for Invar, ${ }^{24}$ which is much lower than those of most conventional metals and closer to that of fused silica, ${ }^{9}$ an Invar sheet from Electronic Space Products International, Inc., was selected to be the cantilever beam. The beam was processed by cutting, polishing, and annealing. The inner diameter of the hollow fused-quartz tubing was chosen to be $132 \mu \mathrm{m}$. A Servometer, Inc., PN-231 regular nickel bellows was used for packaging the flow sensor. The size of the Invar cantilever beam was selected as $3 \mathrm{~mm} \times 30 \mathrm{~mm}$. Based on the working range and resolution of the multimode white-light system and the thickness of the Invar sheet, the gauge lengths and the original air gaps of both fiber sensors can be adjusted to maximize the fringe visibility of both sensors throughout the measurement. Also, the

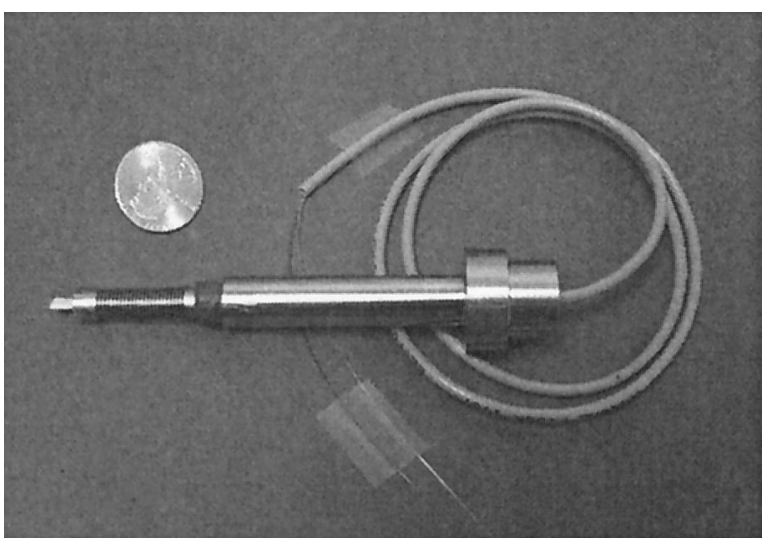

Fig. 4. Prototype fiber optic flow sensor.

gauge length was made as small as possible to decrease the inherent thermal expansion. In the sensor fabrication we adjusted the initial air gap between the end faces of the fibers to obtain the highest interference fringe visibility. A photograph of the finished flow sensor is shown in Fig. 4.

Preliminary experiments on the flow sensor were done in the lab; a 1.5-in. (3.81-cm) lab-scale experimental water-flow testing loop was constructed as shown in Fig. 5. A Flow Technology Company FT24NENW-LEG-5 turbine flowmeter with an accuracy of $\pm 0.2 \%$ for $0-90$ gallons per minute (GPM; 1 gallon $=4.54608 \mathrm{~L}$ ) was used as a reference for calibration of the fiber optic flow sensor. A 0.25 horsepower Wayne submersible utility pump connected with 1.5-in. PVC pipes was used to produce flow rates in the range of $0-18.5$ GPM. Both the fiber optic sensor and the thermometer were inserted into the testing pipes. The fiber optic flow sensor was calibrated by the turbine flowmeter. The sensor construction utilized an Invar cantilever beam with dimensions of $0.25 \mathrm{~mm} \times 38 \mathrm{~mm} \times 3.3 \mathrm{~mm}$. For the front and back sensors (on the two sides of cantilever beam) the original air gaps were 7.21 and $10.13 \mu \mathrm{m}$ and the fringe visibility was $67 \%$ and $51 \%$, respectively. The gauge lengths were 1.8 and $2 \mathrm{~mm}$ and the relative thermal expansion coefficients between the two sensors were 0.9 and 1 , respectively.

The output from the turbine flowmeter and the air gaps from the white-light system are shown in Fig. 6 . It is obvious that the air gaps of the fiber sensors on the two sides of the Invar cantilever beam change in opposite directions as the flow rate changes. The

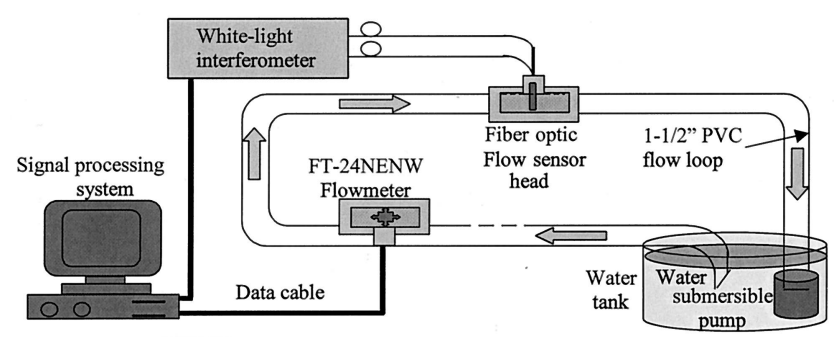

Fig. 5. Lab-scale flow-testing setup. 


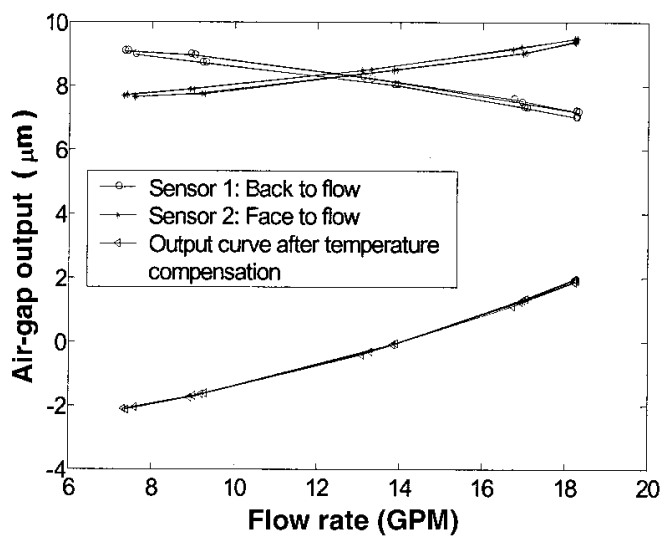

Fig. 6. Self-compensation of the fiber optic flow sensor.

sensors were tested four times with temperature changes from $20^{\circ} \mathrm{C}$ to $45^{\circ} \mathrm{C}$. Although the air-gap changes in the two sensors were affected by temperature, the output of the sensor system compensates for this effect automatically, which verified the theoretical analysis shown in Section 2. Based on the measurement of the sensor output at different flow rates and temperatures, we calibrated the fiber optic flow sensor to obtain the average calibration curve for the measurements. The measurement results at different temperatures are shown in Fig. 7. The resolution of this system is $0.11 \%$, and the repeatability is $\pm 1.62 \%$. We further tested the system by keeping the flow rate steady at 7.12 GPM for $12 \mathrm{~h}$. The system was stable within 0.35 GPM, which corresponds to $1.89 \%$ of the full range 18.4 GPM. We believe that the resolution of the system was limited by the resolution of the reference flowmeter.

\section{Field Testing and Discussion}

To determine the feasibility of on-site flow-rate detection we field tested the flow sensor system at flow loops at the University of Tulsa, Tulsa, Oklahoma. Two flow loops were used in these field tests.

The indoor flow loop, shown in Fig. 8, consists of a variable-frequency pump $(0-1745$ rotations $/ \mathrm{min})$ from Robins \& Meyers, Inc., connected to 1.5-in.

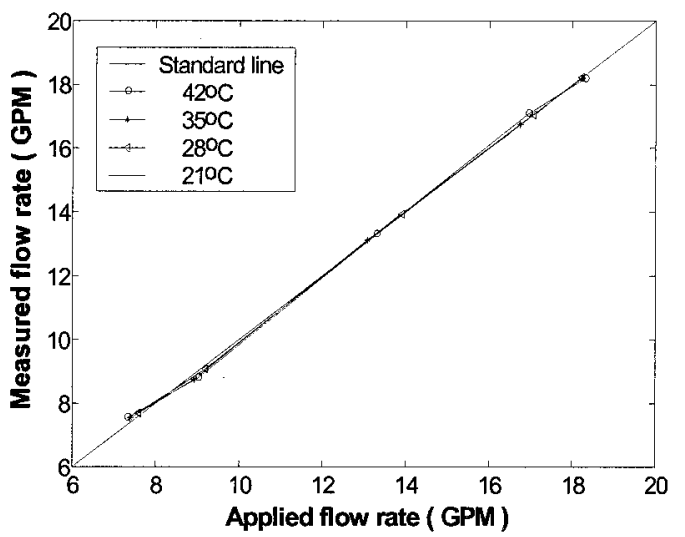

Fig. 7. Results of lab-scale measurement of the fiber optic flow sensor.

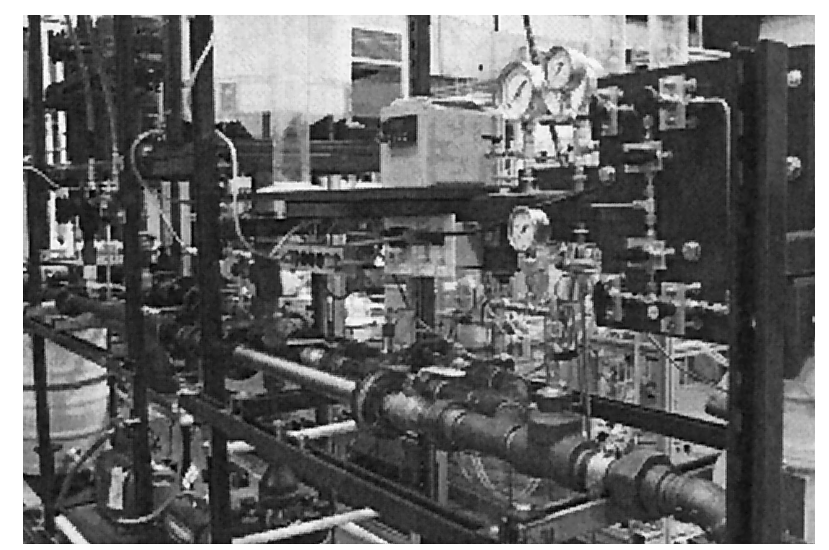

Fig. 8. Indoor flow facilities at Tulsa University, Tulsa, Oklahoma.

Schedule 40 flow loops, which combine a pressureadjustable valve, a pressure gauge from Wika, Inc., and a thermocouple from Omega, Inc. The sensor was deployed in the flow pipes by a $0.5-1.5$-in. $N-P-T$ bushing into a 1.5 -in. $N-P-T$ T fitting, as shown in Fig. 9. The temperature of the flow loops during indoor testing was stable near $29^{\circ} \mathrm{C}$; the pressure in the flow pipes was controlled to $\sim 5$ psi. For the

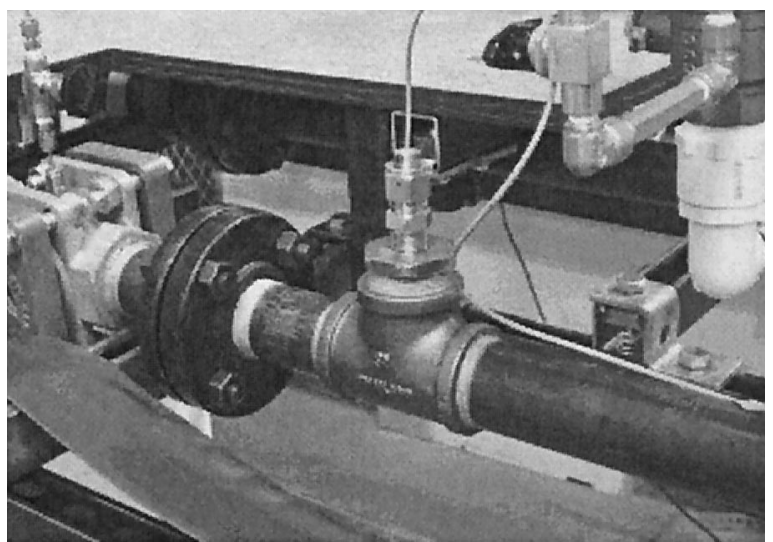

Fig. 9. Fiber flow sensor deployed in the indoor flow loop at Tulsa University.

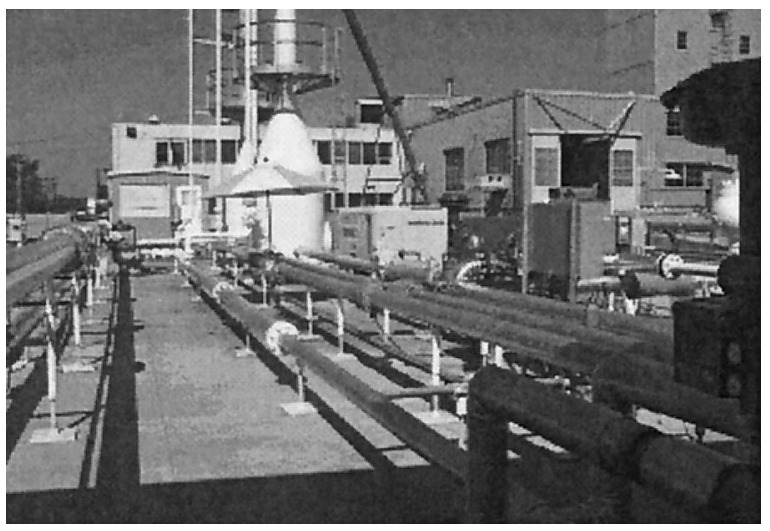

Fig. 10. Outdoor flow facilities at Tulsa University. 


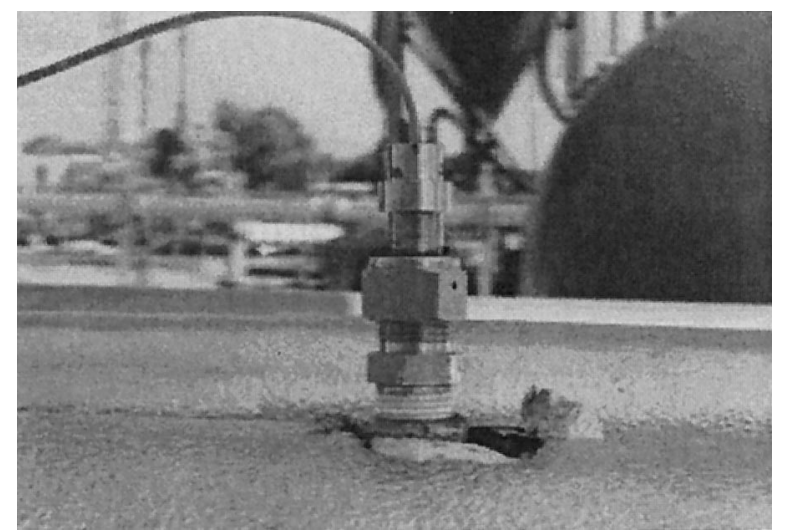

Fig. 11. Fiber sensor deployed in the outdoor flow loop at Tulsa University.

indoor testing the original air gaps of the front and back sensors mounted on the two sides of the cantilever beam of the flow sensor were 10.46 and 11.96 $\mu \mathrm{m}$, the gauge lengths were 1.8 and $2.1 \mathrm{~mm}$, their relative thermal effect coefficients were 0.85 and 1 , and the visibilities were $71 \%$ and $21 \%$, respectively.

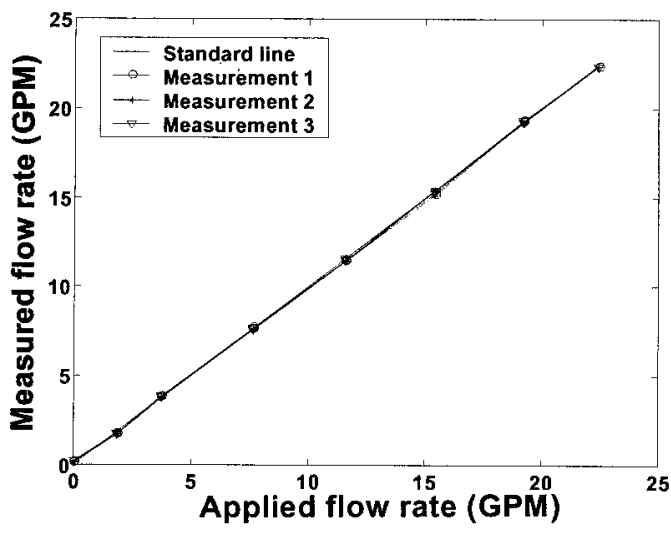

(a)

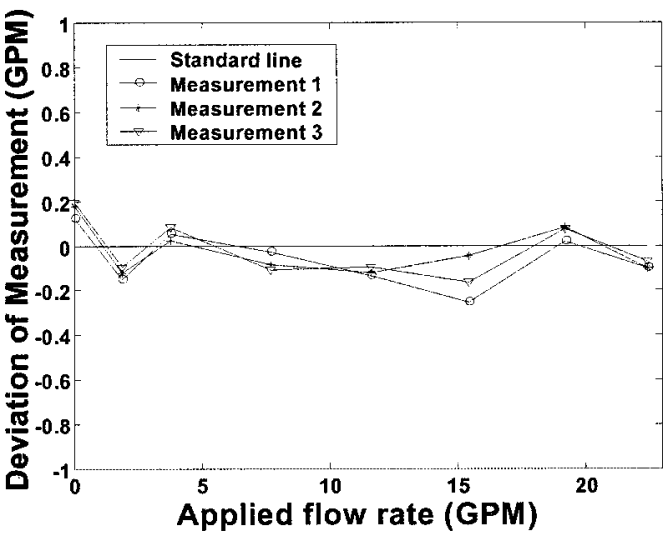

(b)

Fig. 12. (a) Results of flow sensor measurement in the indoor flow loop. (b) Enlargement of the results of repeatability testing of the flow sensor in the indoor flow loop.

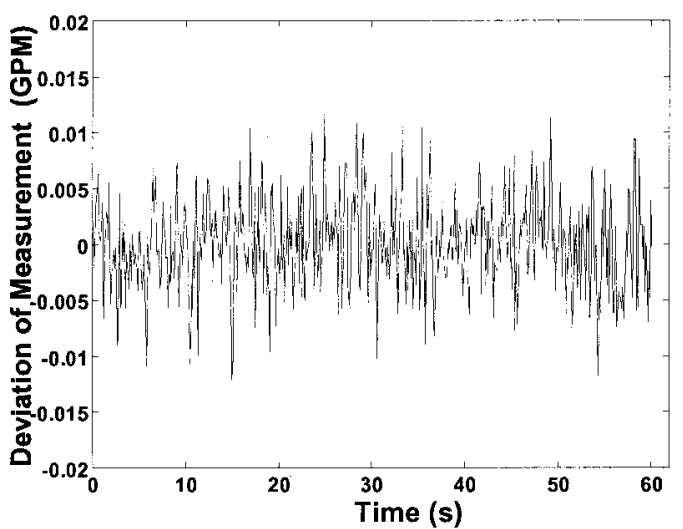

Fig. 13. Resolution of the flow sensor in the indoor flow loop.

As shown in Fig. 10, the outdoor flow loop is a simulated oil field flow environment that combines pipes with diameters of $3-6$ in. at various stages. A variable-frequency pump $(0-2000$ GPM $)$ from Waukesha, Inc., was used to pump water from a 3500-gallon tank. A Micro Motion vibration flowmeter was used as the reference for calibration. Also,

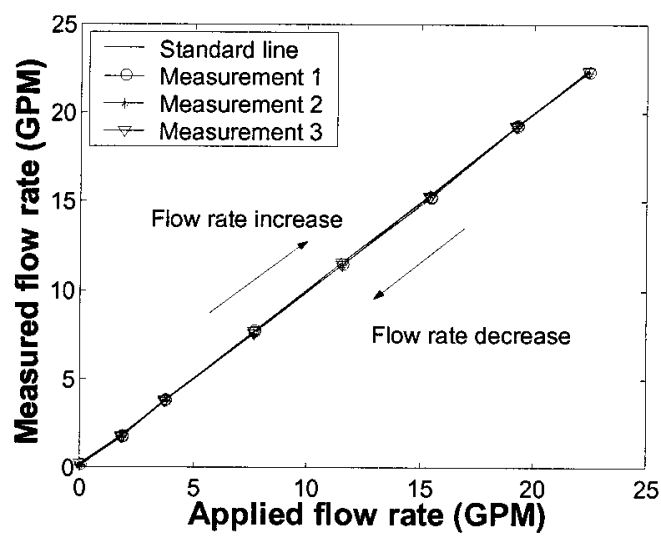

(a)

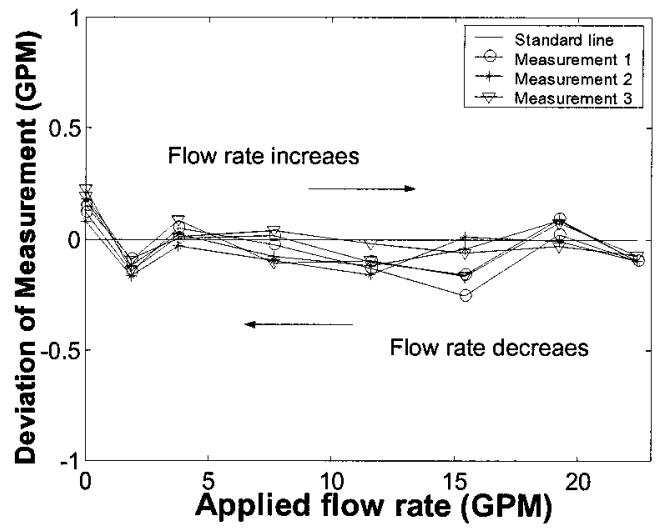

(b)

Fig. 14. (a) Results of hysteresis testing of the flow sensor in the indoor flow loop. (b) Enlargement of the results of hysteresis testing of the flow sensor in the indoor flow loop. 


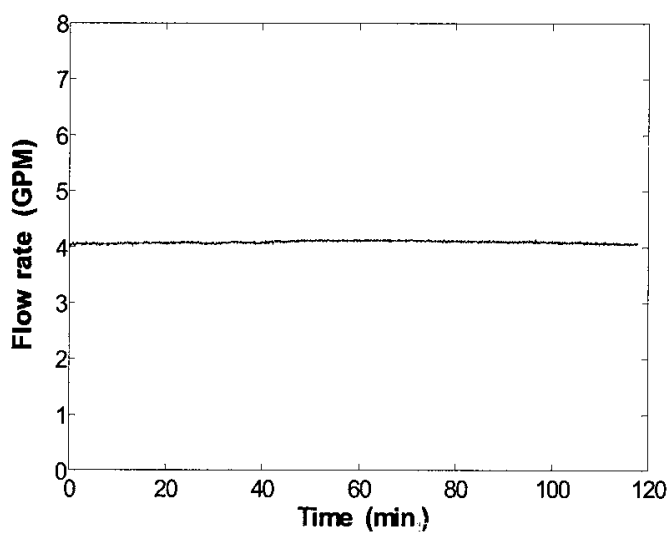

Fig. 15. Stability of the flow sensor in the indoor flow loop.

pressure and temperature gauges from Rosemount, Inc., are were used for monitoring the environmental conditions. A central computer controlled the entire facility, including gauges. As shown in Fig. 11, the fiber optic flow sensor was deployed in the outdoor 3 -in. flow pipes by a $1.5-0.75$-in. $N-P-T$ bushing through into a 0.75 -in. welding port. The sensor probe was connected to the optoelectronics unit in the control room through a dual multimode fiber cable. Using the Micro Motion flowmeter as a reference, we calibrated and tested the sensor system with water circulating in the flow loop at different flow rates. After spectrum detection and signal processing, the output flow-rate data were digitized, displayed, and recorded. The outdoor flow loop testing system experienced pressure fluctuations of approximately 9-66 psi and temperature changes of approximately $26-37^{\circ} \mathrm{C}$. The sensor reported here for outdoor testing was based on a $0.3 \mathrm{~mm} \times 38 \mathrm{~mm} \times 3.3 \mathrm{~mm}$ Invar cantilever beam. For the front and back sensors on the two sides of the cantilever the original air gaps were 9.27 and $9.61 \mu \mathrm{m}$ and the fringe visibility was $52 \%$ and $29 \%$, respectively; both gauge lengths were $1.7 \mathrm{~mm}$, and their relative thermal effect coefficients were the same, set as 1 .

Because of the different lab-scale-testing and fieldtesting conditions, the sensor required recalibration before testing in these field-testing loops. As de-

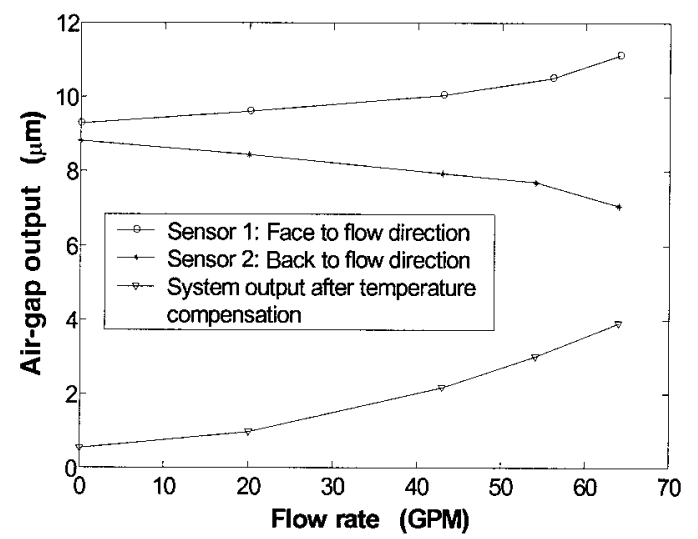

Fig. 16. Output of the flow sensor in the outdoor flow loop.

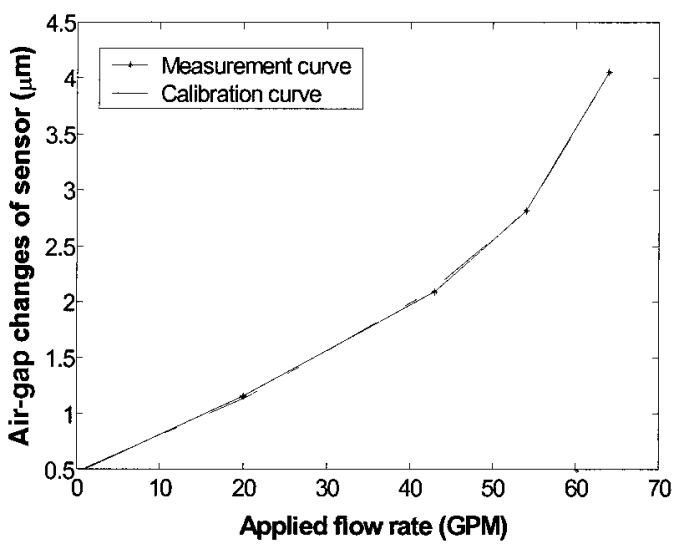

Fig. 17. Calibration curve of the flow sensor for the outdoor flow loop.

scribed in Section 3, compared with the outdoor flow loop, the indoor flow testing facilities had stabler temperature and pressure. Therefore it was not necessary to compensate for temperature and pressure fluctuations; hence, use of only one of the two sensor signals was necessary to characterize the flow rate of the fluid.

The real-time flow-rate information was obtained through the calibrated relationship between the reference flow rate and the change in the air gap of the sensor head. Figure 12(a) shows the measurement results of repeatability testing. As shown in the figure, the flow sensor output is a linear function of the variable-frequency pump's rotational speed. A magnified view of the repeatability results is shown in Fig. 12(b). For the full measurement range from 0 to 22.42 GPM the maximum deviation was \pm 0.26 GPM, which is $\pm 1.16 \%$ of full scale. The resolution of the sensor system is usually represented by twice the standard deviation of a series of flow measurements at a constant flow rate. The sensor resolution was evaluated by use of a calibrated sensor at zero flow rate.

For the indoor flow sensor system, 600 data points were acquired in $1 \mathrm{~min}$ with a sample frequency of 10 counts/s. As shown in Fig. 13, the resolution testing was estimated to be 0.0246 GPM. The normalized resolution with respect to the dynamic range of the system was $0.11 \%$ of the full scale. Also, hysteresis
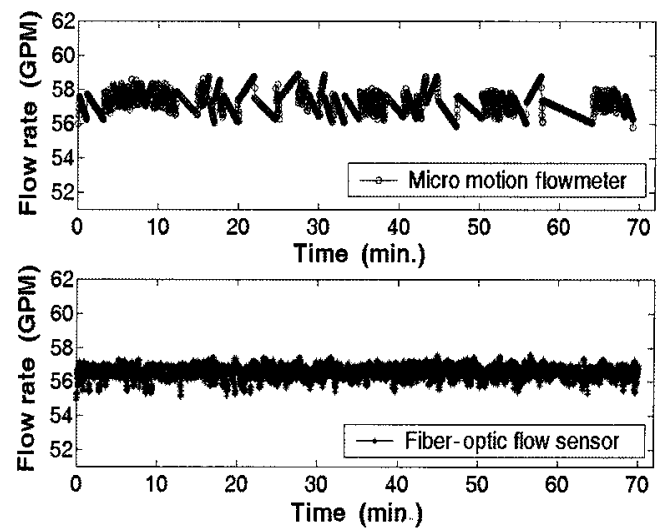

Fig. 18. Stability of flow sensor testing in the outdoor flow loop. 


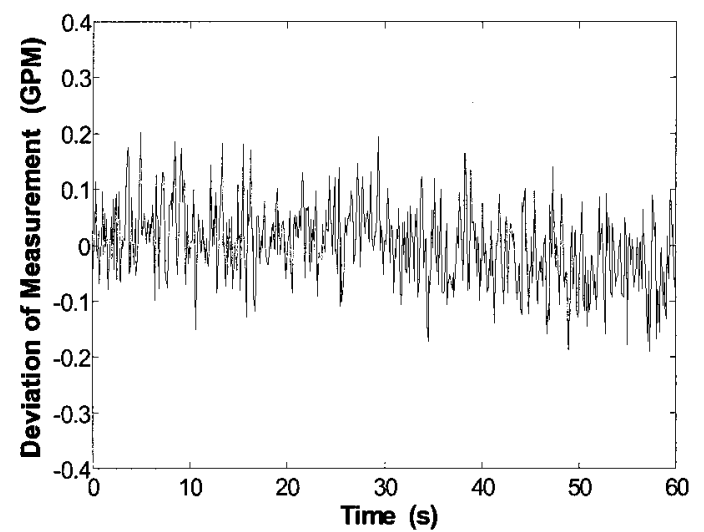

Fig. 19. Resolution of the flow sensor system in the outdoor flow loop.

was tested, as shown in Fig. 14(a). No obvious hysteresis was observed for the measured range, as shown by the enlarged data in Fig. 14(b). The hysteresis was measured to be $\pm 0.89 \%$ of full measurement range for this flow sensor.

To further evaluate the sensor performance in the indoor flow loop, we tested the stability of the sensor

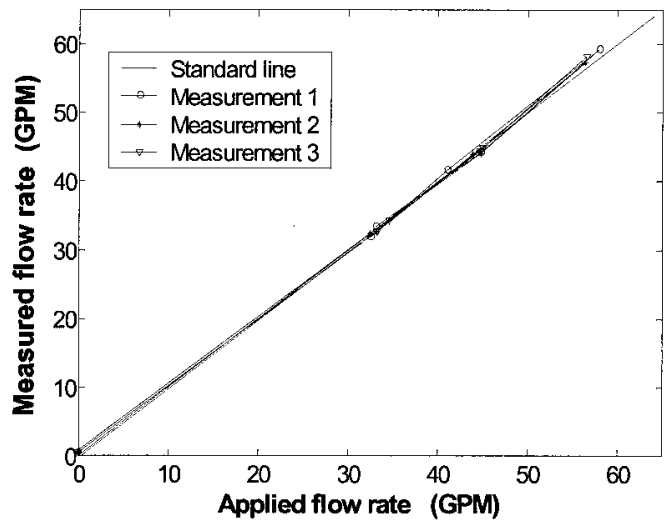

(a)

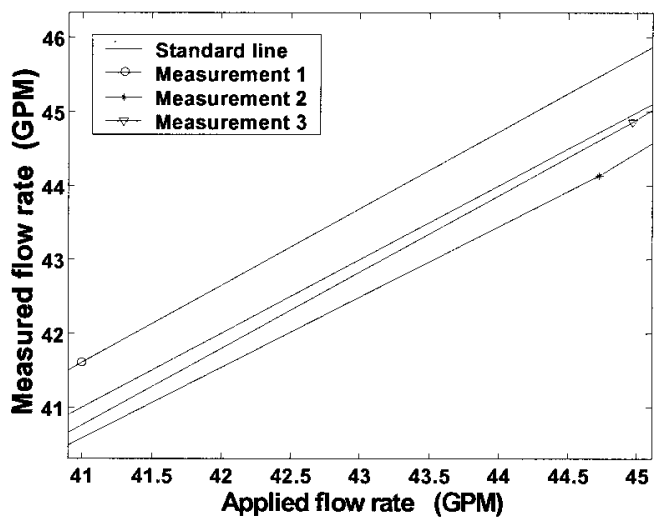

(b)

Fig. 20. (a) Results of flow sensor measurement in the outdoor flow loop. (b) Enlargement of the results of repeatability testing of the flow sensor in the outdoor flow loop. system, and the results are shown in Fig. 15. With the flow rate maintained at 4 GPM for $2 \mathrm{~h}$, and for the fiber optic flow-sensing system measured within $\pm 0.15 \mathrm{GPM}$, the stability of the system was $\pm 0.67 \%$ of the full range over the 2-h period.

For the outdoor testing system, as we mentioned above, because of the complex structure of the outdoor flow-testing system, for each testing step a minimum of $20 \mathrm{~min}$ would be required for a relatively stable flow rate to be obtained. The flowmeter and the pump were designed for a flow-rate range of 0-2000 GPM, with $\pm 0.2 \%$ accuracy of the full range at $\pm 4 \mathrm{GPM}$. So, at the relatively low flow-rate range 0-64 GPM, the accuracy of the reference flowmeter was diminished. When the pump was set at a certain value, the flow rate fluctuated significantly, affecting the evaluation of the fiber optic sensor system's performance. The flow sensor was calibrated and tested based on the average outputs of the Micro Motion flowmeter. Figure 16 shows the air gaps of the two sensors and the output of the system with changes in the flow rate during the calibration. The calibration curve, generated as shown in Fig. 17, provided the coefficient compensation and lineariza-

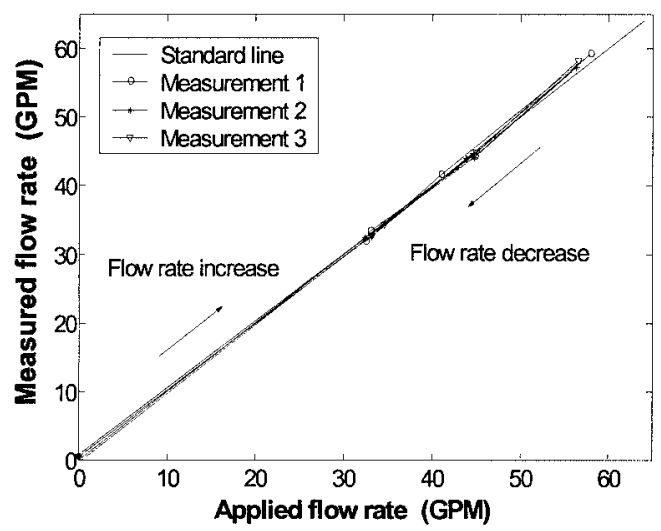

(a)

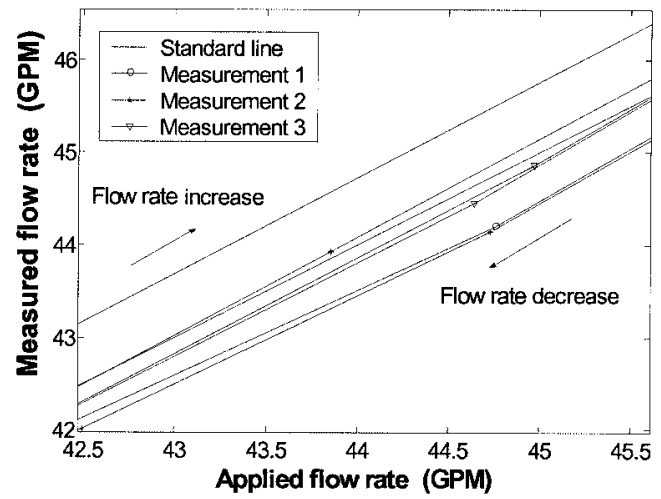

(b)

Fig. 21. (a) Results of hysteresis testing of the flow sensor in the outdoor flow loop. (b) Enlargement of the results of hysteresis testing of the flow sensor in the outdoor flow loop. 
tion. The stability of both the flow sensor and the flowmeter is shown in Fig. 18. Maintaining the variable-frequency pump at $57 \mathrm{GPM}$ for $70 \mathrm{~min}$ yielded a maximum fluctuation of the reference flowmeter of 3.32 GPM, which was greater than that of the fiber optic flow sensor, which had a maximum fluctuation of 3.15 GPM. Also, the standard deviation of the reference flowmeter was 0.61 GPM, whereas that of the fiber optic flow sensor was 0.42 GPM. Based on above data, we believe that the standard deviation of the fiber flow sensor was less than 1 GPM and the stability of this sensor system was better than $1.56 \%$ for a $0-64-G P M$ full scale. The resolution of the fiber optic sensor system for outdoor testing was estimated to be 0.1664 GPM, as shown in Fig. 19. The normalized resolution for this sensor system was $0.26 \%$ of the full scale. Figure 20(a) shows the measurement results of three repetitions of increasing and decreasing flow-rate measurements. The maximum deviation, shown enlarged in Fig. 20(b), was \pm 0.80 GPM, which is $\pm 1.25 \%$ of full scale. Also, as shown in Fig. 21(a), the hysteresis was evaluated as $2.1 \%$ of the full range shown in Fig. 21(b).

\section{Conclusions}

We have described the design, development, and evaluation of a cantilever-beam-based interferometric fiber optic sensor system for single-phase fluid flow-rate measurement. Compared with conventional flow sensors, this fiber optic sensor has the advantages that it is not electrically conducting, is immune to electromagnetic interference, and has high accuracy and small size. The stability of the system has been improved by self-compensating for temperature and pressure sensitivity. This improvement allows the flow-rate measurement to be insensitive to temperature and pressure changes. Both lab-scale and field testing clearly demonstrate the feasibility of applying this fiber optic flow sensor system for real-time flow-rate measurements in fluidfilled pipes. The field testing showed that, in the measurement range 0-64 GPM in 3-in. flow loops, the resolution of the system was $0.26 \%$, the repeatability was $\pm 1.25 \%$, and the stability was $1.56 \%$.

This research is funded by the National Petroleum Technology Laboratory, the U.S. Department of Energy, and Chevron Texaco Research \& Technology Company. The authors thank Troy D. Reed, Len Volk, Mike B. Pickel, and Steven Miska of the drill technology group within the Department of Petroleum Engineering, University of Tulsa, Tulsa, Oklahoma, for kindly offering the use their specialized flow facilities and operation systems and for useful discussions and suggestions for the fiber optic flow sensor system.

\section{References}

1. J. Dakin and B. Culshaw, Optic Fiber Sensors: Principles and Components (Artech House, Boston, Mass., 1988).

2. E. Udd, Fiber Optic Sensors: An Introduction for Engineers and Scientists (Wiley, New York, 1991).
3. N. P. Cheremisinoff, Applied Fluid Flow MeasurementFundamentals and Technology (Marcel Dekker, New York, 1979).

4. R. W. Miller, Flow Measurement Engineering Handbook (McGraw-Hill, New York, 1989), pp. 14-16.

5. F. E. Jones, Techniques and Topics in Flow Measurement (CRC, Boca Raton, Fla., 1995).

6. N. P. Cheremisinoff, Flow Measurement for Engineers and Scientists (Marcel Dekker, New York, 1988).

7. J. H. Lyle and C. W. Pitt, "Vortex shedding fluid flowmeter using optic fiber sensor," Electron. Lett. 17, 244-245 (1981).

8. P. A. Leilabady, J. D. C. Jones, A. D. Kersey, M. Corke, and D. A. Jackson, "Monomode fiber optic vortex shedding flowmeter," Electron. Lett. 20, 664-665 (1984).

9. S. Webster, R. McBride, J. S. Barton, and J. D. C. Jones, "Air flow measurement by vortex shedding from multimode and monomode optic fibers," Meas. Sci. Technol. 3, 210-216 (1992).

10. M. J. Weber, Optical Materials (CRC, Boca Raton, Fla., 1995), pp. $72-73$.

11. W. M. Wang, W. J. O. Boyle, K. T. V. Grattan, and A. W. Palmer, "Fiber-optic Doppler velocimeter that incorporates active optical feedback from a diode laser," Opt. Lett. 17, 819821 (1992)

12. S. W. James, R. A. Lockey, D. Egan, and R. P. Tatam, "Fiber optic reference beam laser Doppler velocimetry," Opt. Commun. 119, 460-464 (1995).

13. Y. Imai and K. Tanaka, "Direct velocity sensing of flowing distribution based on low-coherence interferometry," J. Opt. Soc. Am. A 16, 2007-2011 (1999).

14. W. Wang, S. Yee, and P. G. Reinhall, "Fluid viscosity and mass flow sensor using forward light scattering," in Pacific Northwest Fiber Optic Sensor Workshop, E. Udd, ed., Proc. SPIE 2574, 146-151 (1995).

15. J. X. Fang, H. F. Taylor, and H. S. Choi, "Fiber-optic FabryPerot flow sensor," Microwave Opt. Technol. Lett. 18, 209-211 (1998).

16. P. A. Leilabady, J. D. C. Jones, and D. A. Jackson, "Monomode fiber optic interferometric techniques in flow velocity measurement," Opt. Acta 32, 233-240 (1985).

17. R. J. G. Carr, "Fiber optic sensors for the characterization of particles size and flow velocity," Sensors Actuators A 21-23, 1111-1117 (1990).

18. D. Rebiere, C. Dejous, J. Pistre, and J. Aucouturier, "Acoustic wave devices to measure flow: comparison between surface acoustic wave (SAW) and shear horizontal acoustic plate (SHAPM) mode oscillators," Sensors Actuators A 41-43, 384-388 (1994).

19. J. P. Dunkers, J. L. Lenhart, S. R. Kueh, J. H. van Zanten, S. G. Advani, and R. S. Parnas, "Fiber optic flow and cure sensing for liquid composite molding," Opt. Lasers Eng. 35, 91-104 (2001)

20. A. Wang, M. S. Miller, A. J. Plante, M. F. Gunther, K. A. Murphy, and R. O. Claus, "Split-spectrum intensity-based optical fiber sensors for measurement of microdisplacement, strain, and pressure," Appl. Opt. 35, 2595-2601 (1996).

21. A. Wang, H. Xiao, J. Wang, Z. Wang, W. Zhao, and R. G. May, "Self-calibrated interferometric-intensity-based optical fiber sensors," J. Lightwave Technol. 19, 1495-1501 (2001).

22. B. Qi, G. R. Pickrell, J. Xu, P. Zhang, Y. Duan, W. Peng, Z. Huang, W. Huo, H. Xiao, R. G. May, and A. Wang, "Novel data processing techniques for dispersive white light interferometer," Opt. Eng. 42, 3165-3171 (2003).

23. D. B. Marghitu, Materials Engineering Handbook (Academic, San Diego, Calif., 2001), pp. 131-135.

24. G. S. Brady, H. R. Clanser, and J. A. Vaccari, Materials Handbook (McGraw-Hill, New York, 1997), p. 518. 